\title{
Transport and Noise Properties of sub-100-nm Planar Nb Josephson Junctions with Metallic Hf-Ti Barriers for nano-SQUID Applications
}

\author{
V. Morosh, ${ }^{1}$ J. Linek, ${ }^{2}$ B. Müller, ${ }^{2}$ M.J. Martínez-Pérez $\odot,{ }^{2, \dagger}$ S. Wolter, ${ }^{1}$ T. Weimann, ${ }^{1}$ J. Beyer, ${ }^{3}$ \\ T. Schurig, ${ }^{3}$ O. Kieler, ${ }^{1}$ A.B. Zorin $\odot,{ }^{1}$ R. Kleiner, ${ }^{2}$ and D. Koelle $\odot^{2, *}$ \\ ${ }^{1}$ Department Quantum Electronics, Physikalisch-Technische Bundesanstalt (PTB), Bundesallee 100, 38116 \\ Braunschweig, Germany \\ ${ }^{2}$ Physikalisches Institut, Center for Quantum Science (CQ) and LISA ${ }^{+}$, University of Tübingen, Auf der \\ Morgenstelle 14, 72076 Tübingen, Germany \\ ${ }^{3}$ Department Cryophysics and Spectrometry, Physikalisch-Technische Bundesanstalt (PTB), Abbestraße 2, \\ 10587 Berlin, Germany
}

(Received 11 March 2020; revised 2 October 2020; accepted 6 November 2020; published 30 November 2020)

\begin{abstract}
We analyze electric transport and noise properties at $4.2 \mathrm{~K}$ of self-shunted superconductor-normal metal-superconductor (SNS) sandwich-type Josephson junctions, comprising $\mathrm{Nb}$ as the superconductor and Hf-Ti as the normal conducting material, with lateral dimensions down to approximately $80 \mathrm{~nm}$. The junctions are fabricated with an optimized multilayer $\mathrm{Nb}$ technology based on nanopatterning by electron-beam lithography and chemical-mechanical polishing. The dependence of transport properties on the junction geometry (lateral size and barrier thickness $d_{\mathrm{Hf}-\mathrm{Ti}}$ ) is studied, yielding a characteristic voltage $V_{c}$ up to approximately $100 \mu \mathrm{V}$ for the smallest $d_{\mathrm{Hf}-\mathrm{Ti}}=17 \mathrm{~nm}$. The observed small hysteresis in the current-voltage curves of devices with high $V_{c}$ and large size can be attributed to self-heating of the junctions and fitted with an extended version of the resistively shunted junction model. Measurements of voltage noise of single junctions are consistent with the model including self-heating effects. The potential of our technology for further miniaturization of nanoscale superconducting quantum interference devices and for the improvement of their performance is discussed.
\end{abstract}

DOI: 10.1103/PhysRevApplied.14.054072

\section{INTRODUCTION}

Superconducting quantum interference devices (SQUIDs) are an important application of overdamped Josephson junctions (JJs) [1,2]. Recently, there has been a clear trend towards miniaturization of these very sensitive magnetic flux detectors, with the goal to make them suitable for investigations of nanomagnetism [3-11], possibly up to strong magnetic fields in the tesla range [12-15]. The fields produced by magnetic nanoparticles (MNPs) are of magnetic dipole type and, therefore, decay rather quickly (cubically) with distance. To ensure maximum coupling of such a dipole to a submicrometer-sized SQUID (nanoSQUID) the latter should be positioned as close as possible to the dipole and have small component sizes (JJ size, loop area, width and thickness of the leads) [4]. Another important reason for shrinking the dimensions of the SQUID is the need to avoid penetration of Abrikosov vortices into the superconducting thin film structures [16-18] and of

\footnotetext{
*koelle@uni-tuebingen.de

†Present address: Instituto de Ciencia de Materiales de Aragon and Departamento de Fisica de la Materia Condensada, CSICUniversidad de Zaragoza, 50009 Zaragoza, Spain.
}

Josephson vortices into the junction barriers, which may occur when a strong external magnetic field is applied for manipulation of the magnetic moment of a MNP. Therefore, suitable JJs should be sufficiently small, but still exhibit practical electrical characteristics.

The characteristic voltage

$$
V_{c}=I_{c} R_{n}
$$

of a JJ, with critical current $I_{c}$ and normal resistance $R_{n}$, determines basic dynamic and noise properties of the JJ [19]. Self-shunted superconductor-normal metalsuperconductor (SNS) junctions usually have rather low values of $V_{c}$ due to their small $R_{n}$. The latter feature is in contrast to other conventional overdamped junctions, like Dayem bridges (see, e.g., Ref. [19]), small superconductor-insulator-superconductor (SIS) [20] or intrinsically shunted SNIS JJs [21], which typically have $V_{c}$ of the order of $100 \mu \mathrm{V}$. However, SNS junctions combine a number of crucial advantages, making them suitable for the realization of nano-SQUIDs. Unlike SIS JJs with practically limited critical current density, $j_{c} \equiv I_{c} / A \leq 20 \mathrm{kA} / \mathrm{cm}^{2}$ ( $A$ is the area of the junction) stemming from the formation of unwanted pinholes in very 
thin barriers [22], SNS JJs with barriers made of Pd-Au or Hf-Ti can reach very high $j_{c}$ of several $100 \mathrm{kA} / \mathrm{cm}^{2}$ [23]. This is an important feature, which allows one to keep $I_{c}$ sufficiently high, while reducing the lateral JJ size to sub-100-nm dimensions. From a practical point of view, a miniaturized SNS junction still may have a widely tunable $j_{c}$ by adjusting the normal conducting layer thickness.

Moreover, because of a relatively large barrier thickness (approximately 15-30 nm), small-area SNS JJs have negligible intrinsic capacitance $C$ (even if we assume a rather large value of permittivity $\varepsilon$ that may occur in SNS JJs with a $\mathrm{Nb}$-Si barrier $[24,25])$, i.e., the Stewart-McCumber parameter [26,27] $\beta_{C}=\left(2 \pi I_{c} R_{n}^{2} C\right) / \Phi_{0} \ll 1$, where $\Phi_{0}$ is the magnetic flux quantum. Parasitic capacitance, originating from overlapping parts of superconducting electrodes is usually small, too. Therefore, the current $I$ versus voltage $V$ curves ( $I-V$ curves) should be free of hysteresis related to capacitance. The nonhysteretic $I-V$ curve is then well described by the simple square root expression derived within the framework of the resistively shunted junction (RSJ) model (for negligible thermal noise) [28]

$$
V=R_{n}\left(I^{2}-I_{c}^{2}\right)^{1 / 2} \quad \text { for } I>I_{c} .
$$

The use of electron-beam lithography (EBL) for nanopatterning provides high spatial resolution, even in the deepsubmicron regime. Hence, good reproducibility of device geometry can be achieved even for sub-100-nm SNS JJs. Even some deviations from the nominal shape and size, which can occur during the fabrication of very small JJs, do not change the transport parameters substantially. In contrast, the properties of superconducting weak links of Dayem bridge type undergo a significant departure from the target parameters, if a tiny deviation from the designed shape or size of the constriction appears [7]. Moreover, these weak links (especially those having a twodimensional architecture) suffer from appreciable thermal instability due to large power dissipation and inefficient heat drain from the small constriction. This leads to the undesirable, so-called thermal hysteresis in the $I-V$ curve $[29,30]$.

There have been several earlier realizations of nanoSQUIDs based on self-shunted SNS JJs with Hf-Ti barrier and linear sizes appreciably larger than $100 \mathrm{~nm}$. Because of their low-noise performance and relatively compact design, nano-SQUIDs based on these junctions promise great potential as stand-alone devices $[31,32]$ and as building blocks of complex circuits $[33,34]$. However, there is still a need for further improvements regarding size reduction of the SQUIDs and tuning of their electrical parameters, including $I_{c}, R_{n}$, and, therefore, $V_{c}$.

In this paper, we introduce a $\mathrm{Nb}$ planar technology optimized for fabrication of ultra-small JJs with Hf-Ti barrier thicknesses from 17-26 nm. We study the transport properties of these sandwich-type junctions with lateral dimensions down to approximately $80 \mathrm{~nm}$ and different values of $j_{c}$ and $V_{c}$. Their transport and noise properties are compared to JJs with larger size of up to $1 \mu \mathrm{m}$. We discuss the potential for improving the performance of nano-SQUIDs containing such junctions. This includes possible minimization of the sensor size and improving the flux noise properties.

\section{FABRICATION TECHNOLOGY AND GEOMETRY OF SMALL JUNCTIONS}

Our planar fabrication technology based on EBL and chemical-mechanical polishing (CMP), utilizing $\mathrm{Nb}$ as the superconductor and $\mathrm{Hf}_{\mathrm{wt}} 50 \% \mathrm{Ti}_{\mathrm{wt}} 50 \%$ as the normal conducting compound barrier [23], has successfully been employed for the fabrication of pulse-driven ac voltage standard circuits [35]. The composition $\mathrm{Hf}_{\mathrm{wt}} 50 \% \mathrm{Ti}_{\mathrm{wt}} 50 \%$ is chosen to maximize the resistivity $\rho_{\mathrm{Hf}-\mathrm{Ti}}$ of the normal conducting layer [23]. Typical junction size in these circuits is in the range between $0.5 \mu \mathrm{m} \times 0.5 \mu \mathrm{m}$ and $1 \mu \mathrm{m} \times 1 \mu \mathrm{m}$, and the characteristic voltage at temperature $T=4.2 \mathrm{~K}$ is at moderate values of $V_{c} \approx 50 \mu \mathrm{V}$. Recently, we optimized this technology for the fabrication of sub-100-nm SNS JJs with possibly well-defined shapes and reproducible parameters, including as high as possible $V_{c}$.

For this purpose, fabrication parameters including etching, polishing, EBL exposure and developing throughout the wafer processing have been optimized. Moreover, $\mathrm{CF}_{4}$ normally used in the original process for reactive ion etching (RIE) of the $\mathrm{Nb}$ top electrode is replaced by inductively coupled plasma (ICP) RIE with $\mathrm{SF}_{6}$, providing higher etching rates and better anisotropy. This method substantially improves the steepness of sidewalls and allows for better control of the electrode cross-section dimensions. Another peculiarity of the optimized process is the application of the high-resolution negative resist ARN 7520.18 to shape the bottom $\mathrm{Nb}$ wiring. This allows us to put approximately $100-\mathrm{nm}$ narrow $\mathrm{Nb}$ current lines as close as $70-100 \mathrm{~nm}$ to each other. Altogether, the increased steepness of sidewalls (approaching $90^{\circ}$ ) and closer arrangement of structures leads to a more compact geometry of the circuit, allowing for a potentially higher density of elements on a chip.

The fabrication process is sketched in Fig. 1 in a simplified form. A thermally oxidized $\mathrm{Si}$ wafer with a native $300-$ nm-thick $\mathrm{SiO}_{2}$ layer is utilized as the substrate. The process starts with a precleaning step and the magnetron sputter deposition (MSD) of a 30-nm-thick $\mathrm{Al}_{2} \mathrm{O}_{3}$ layer (etch stop layer), followed by the MSD of a $\mathrm{Nb} / \mathrm{Hf}-\mathrm{Ti} / \mathrm{Nb}$ trilayer (with thicknesses $160-250 \mathrm{~nm} / 17-26 \mathrm{~nm} / 150-200$ $\mathrm{nm}$, respectively) in situ in a high-vacuum chamber at room temperature [Fig. 1(a)]. Next, the JJs are formed using an approximately 30 -nm-thick Al hard mask patterned by EBL and following lift-off. Uncovered $\mathrm{Nb}$ is 
(a)

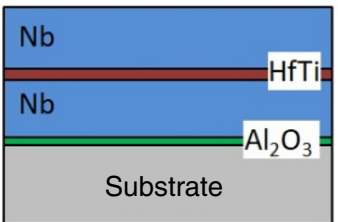

(c)

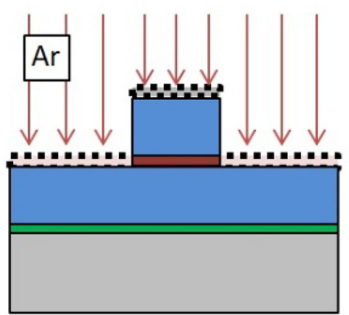

(e)

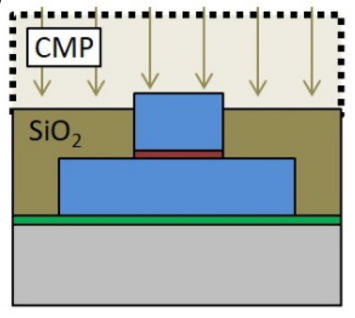

(b)

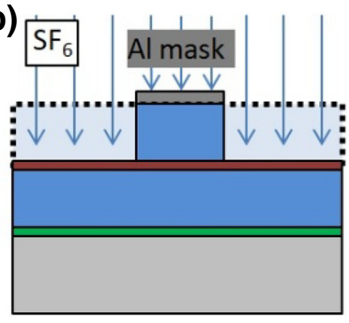

(d)

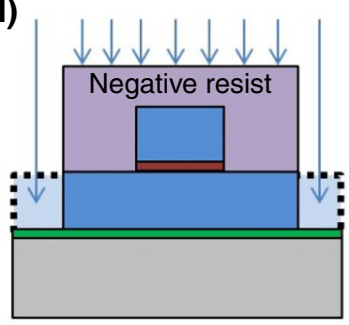

(f)

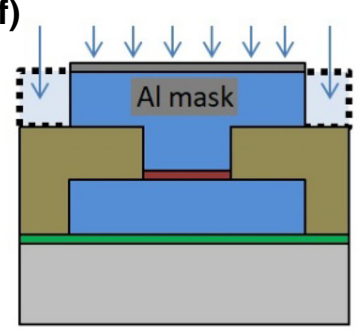

(g)

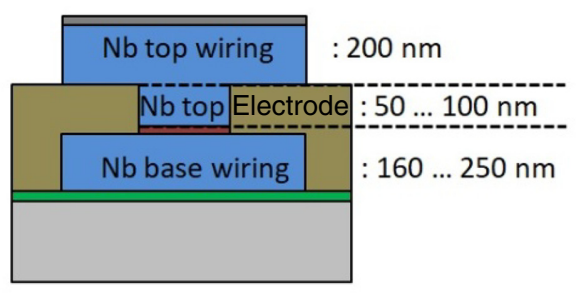

FIG. 1. Simplified layer scheme showing key steps of the SNS JJ fabrication technology: (a) $\mathrm{Al}_{2} \mathrm{O}_{3}$ etching stop layer and initial trilayer on a Si wafer; (b) ICP RIE of the $\mathrm{Nb}$ top layer covered by a prepatterned $\mathrm{Al}$ hard mask; (c) simultaneous Ar-ion milling of the barrier material and Al hard mask; (d) ICP RIE of the bottom $\mathrm{Nb}$ wiring patterned by high-resolution resist; (e) polishing of a $\mathrm{SiO}_{2}$ layer preliminary created by PECVD; (f) ICP-RIE patterning of the $\mathrm{Nb}$ top wiring using an $\mathrm{Al}$ hard mask; (g) final device; numbers indicate thickness of the $\mathrm{Nb}$ layers.

removed by ICP RIE [Fig. 1(b)] and, afterwards, the Hf$\mathrm{Ti}$ barrier and the $\mathrm{Al}$ hard mask are simultaneously etched via Ar-ion beam milling [Fig. 1(c)]. Then, the bottom $\mathrm{Nb}$ wiring layer is patterned by a high-resolution negative resist mask prepared by EBL and ICP RIE [Fig. 1(d)]. The plasma enhanced chemical vapor deposition (PECVD) of an approximately 600 -nm-thick $\mathrm{SiO}_{2}$ layer and subsequent CMP are performed both to planarize the surface and to uncover the top electrode for an electric contact to the following $\mathrm{Nb}$ layer [Fig. 1(e)]. This layer, which is deposited

via the MSD and subsequently patterned using an $\mathrm{Al}$ hard mask formed by EBL and ICP RIE, serves as the top $\mathrm{Nb}$ wiring [Fig. 1(f)]. In Fig. 1(g) we show the final structure of the device.

Small departures from the nominal feature sizes can occasionally occur during EBL and etching. In the case of JJs with relatively large lateral sizes (typically greater than $500 \mathrm{~nm}$ ), this results in a negligible spread of JJ sizes. In contrast, the smaller JJs are much more sensitive to uncertainties in the definition of the junction area. Thus, a systematic study of JJ parameters on a variety of junctions differing in size is necessary. We fabricated JJs with lateral size down to about $80 \mathrm{~nm}$. The departure of the $\mathrm{JJ}$ size and shape from nominal dimensions is investigated in detail for square-shaped JJs with nominal width $w_{n}$ ranging from $50 \mathrm{~nm}$ to $1 \mu \mathrm{m}$ and with seven different values for the barrier thickness $d_{\mathrm{Hf}-\mathrm{Ti}}$ between $17 \mathrm{~nm}$ and $26 \mathrm{~nm}$ (fabricated from seven different wafers).

A systematic enlargement (up to an extra $50 \mathrm{~nm}$ ) with respect to the designed JJ width is caused by the proximity effect occurring during the electron-beam exposure. A statistical deviation in the $\mathrm{JJ}$ width of maximum \pm 20 $\mathrm{nm}$ is estimated from scanning electron microscopy (SEM) images. Additionally, due to the above-mentioned proximity effect, the resulting JJ area takes a shape with notably rounded corners.

JJs designed with $w_{n}=50 \mathrm{~nm}$ are converted into almost circular-shaped JJs with diameter approximately $(80 \pm 10)$ $\mathrm{nm}$ [see Figs. 2(a)-2(c)]. For larger JJs, we observe rounding of the corners with a curvature radius $R \sim 40 \mathrm{~nm}$ [Fig.
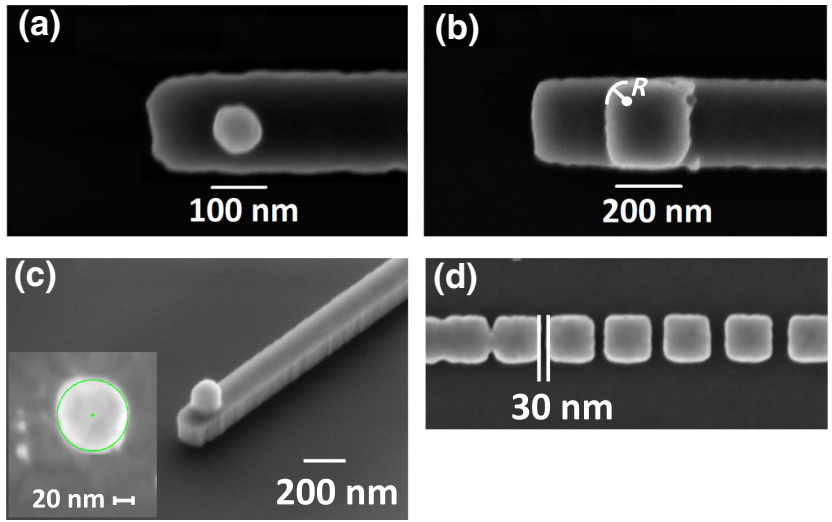

FIG. 2. SEM images of fabricated device structures. Panels (a) -(c) show top electrodes and base wiring [after step (d) in Fig. 1]: (a) JJ with $w=50 \mathrm{~nm}$ (designed as a square-shaped junction) has a circular shape with diameter $(80 \pm 10) \mathrm{nm}$; (b) JJ with $w=200 \mathrm{~nm}$ has an actual width $(230 \pm 10) \mathrm{nm}$ and rounded corners with radius $R \sim 40 \mathrm{~nm}$; (c) $45^{\circ}$ tilted view of a JJ with $w=50 \mathrm{~nm}$, showing steep sidewalls; inset shows top view of the Al mask after lift-off [step (b) in Fig. 1] with $80 \mathrm{~nm}$ diameter (green circle). (d) Test structure with variable spacing between top electrodes [after step (c) in Fig. 1] showing the shortest distance between adjacent JJs of approximately $30 \mathrm{~nm}$. 
2(b)]. Therefore, the effect of rounding of the corners and some increase of the junction area (as compared to the nominal JJ size) has to be considered for a precise design of circuits that contain such ultra-small JJs.

For creating nano-SQUIDs with ultra-small size of the out-of-plane loop [33], two JJs have to be placed as close as possible to each other. To explore this limit, we fabricated test structures that contain arrays of free-standing unwired JJs with variable spacing between them. As shown in Fig. 2(d), we achieved the shortest distance of about $30 \mathrm{~nm}$ between two JJs that still ensures electric isolation of the JJs. More dense arrangements of elements and their ultimate dimensions will require appropriate tuning of the lithographic steps (i.e., resist thickness, developing parameters, etc.), hard mask lift-off, and etching and deposition times.

\section{CURRENT-VOLTAGE CHARACTERISTICS}

A set of representative $I-V$ characteristics of $w_{n}=50$ and $200 \mathrm{~nm} \mathrm{JJs}$, with different barrier thicknesses and, therefore, different $j_{c}$ is shown in Figs. 3(a) and 3(b), respectively. The $I-V$ curves are measured at $T=4.2 \mathrm{~K}$ in a current bias mode.

The RSJ model with zero junction capacitance $\left(\beta_{C}=0\right)$ and zero noise $(T=0)$ is applied for fitting [with Eq. (2)] those experimental $I-V$ curves that reveal a linear behavior in the voltage state not too far above $I_{c}$, and that do not show any trace of hysteresis (Fig. 3 dashed lines). The fits at low currents are in good agreement with the measurement. At high currents $\left(I \gtrsim 2 I_{c}-4 I_{c}\right.$ depending on the sample) the experimental $I-V$ curves deviate from the linear dependence given by Eq. (2), showing a behavior with continuously rising differential resistance [in Fig. 3(a) the $I-V$ curves of JJs with $d_{\mathrm{Hf}-\mathrm{Ti}}=19.5$ and $\left.21.7 \mathrm{~nm}\right]$. We attribute this to a change in the JJ resistance that is likely caused by appreciable self-heating [37].

Measurements of $\rho_{\mathrm{Hf}-\mathrm{Ti}}(T)$ on bare Hf-Ti thin film stripes reveal $\rho_{\mathrm{Hf}-\mathrm{Ti}} \approx$ const at low $T$, and a continuous increase only above $T \approx 25 \mathrm{~K}$, which is much higher than the $\mathrm{Nb}$ critical temperature $T_{c}(\mathrm{Nb})=9.2 \mathrm{~K}$ as determined from test structures on our wafers. Still, heating of the barrier material only slightly above $T_{c}(\mathrm{Nb})$ might already impact the adjacent superconductor by making a thin $\mathrm{Nb}$ interface layer normal conductive and, therefore, adding to the total JJ resistance. Anyway, the described increase in differential resistance of some of the JJs is observed far from the optimal working point of dc SQUIDs located typically at bias currents per JJ close to $I_{c}[1]$ and hence should not dramatically impact electric properties of SQUIDs for practical applications.

As can be seen from Fig. 3, the $I-V$ curves of the samples with the smallest $d_{\mathrm{Hf}-\mathrm{Ti}}$ exhibit either pronounced hysteresis $\left(w_{n}=200 \mathrm{~nm} \mathrm{JJ}\right.$ with $\left.d_{\mathrm{Hf}-\mathrm{Ti}}=17 \mathrm{~nm}\right)$ or some traces of hysteresis $\left(w_{n}=50 \mathrm{~nm} \mathrm{JJ}\right.$ with $d_{\mathrm{Hf}-\mathrm{Ti}}=17 \mathrm{~nm}$ and
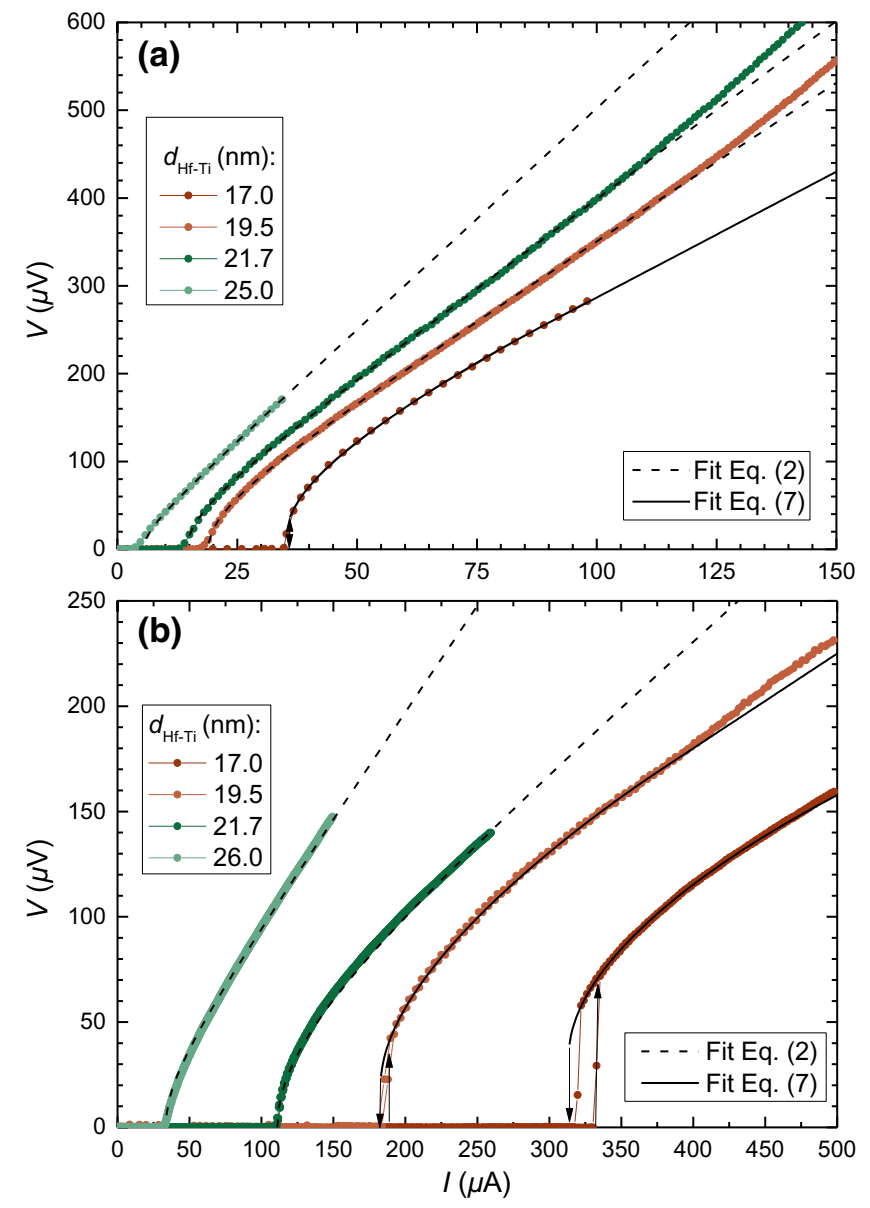

FIG. 3. $I-V$ curves measured at $4.2 \mathrm{~K}$ (symbols) of (a) $w_{n}=$ $50 \mathrm{~nm}$ and (b) $w_{n}=200 \mathrm{~nm}$ JJs with different barrier thicknesses $d_{\text {Hf-Ti }}$. Dashed lines show fits based on the RSJ model Eq. (2). Solid lines show fits based on the extended RSJ model Eq. (7) after Gubankov et al. [36].

$w_{n}=200 \mathrm{~nm} \mathrm{JJ}$ with $d_{\mathrm{Hf}-\mathrm{Ti}}=19.5 \mathrm{~nm}$ ). In the literature, there are two leading models that explain hysteresis in SNS junctions. According to the first model, power dissipation can cause a significant rise in the temperature inside the JJ once it switches to the voltage state. Such a self-heating effect of the junction [36-38] leads to the so-called thermal hysteresis. The second model is based on the assumption of a high effective junction capacitance $C_{\text {eff }}$ (and, thus, $\beta_{C}=$ $2 \pi I_{c} R_{n}^{2} C_{\text {eff }} / \Phi_{0} \gtrsim 1$ ), which may originate from the limited speed of the fundamental energy relaxation mechanism in the normal region [39-41]. However, this mechanism is expected to be relevant for SNS JJs with relatively thick barrier layers, i.e., $d_{\mathrm{Hf}-\mathrm{Ti}} \gg \xi_{S}$ [41], which is not the case in our JJs $\left(\xi_{S}\right.$ is the superconductor coherence length of the superconductor layers).

Moreover, we argue that the parasitic capacitance in our samples, which might originate from peripheral components like current lines, is also not responsible for the formation of the hysteresis. Calculations of the capacitance contribution from overlapping $\mathrm{Nb}$ layers give relatively 
small values, i.e., $C \leq 100 \mathrm{fF}$. However, to explain the observed behavior applying the RCSJ model with finite capacitance, one should put the junction capacitance in the range from $0.4 \mathrm{pF}$ to an enormously large value of $12 \mathrm{pF}$.

We assume that self-heating is the dominant mechanism responsible for hysteresis in our SNS JJs. In the nonzero voltage state the power dissipated in the barrier leads to an elevated temperature $T^{*}$ and, therefore, to reduced values of the critical current (as, e.g., observed in thin-film $\mathrm{Al} / \mathrm{Cu} / \mathrm{Al}$ SNS JJs [38]). Taking into account the balance of currents in the RSJ model, i.e., $I=I_{c} \sin \delta+$ $\left(\Phi_{0} / 2 \pi R_{n}\right)(\partial \delta / \partial t)$, the average value of the oscillating Josephson current $\left\langle I_{c} \sin \delta\right\rangle=I-V / R_{n}$ reduces ( $\delta$ is the gauge-invariant phase difference across the junction). This effect gives rise to lower currents $I$ for given values of $V$ and, hence, to a negative slope region in the $I-V$ curve close to $I_{c}$, which for the bias current sweep in both directions is observed as hysteresis. Such behavior has been modeled by Gubankov et al. [36].

The Gubankov model (see also Sec. V.D.5. of Ref. [19]) makes two simplifying assumptions. Firstly, the power $P=I V$ dissipated in the junction is assumed to cause a linear increase in temperature

$$
\Delta T=T^{*}-T=\kappa P=\kappa I V,
$$

where $T$ is the bath temperature, $T^{*}$ is an elevated temperature of the junction, and the constant $\kappa \equiv \Delta T / P$ is the thermal resistance of the system. Secondly, a simple linear dependence

$$
V_{c}(T)=\alpha\left(T_{c}-T\right)
$$

is assumed, which then yields

$$
V_{c}\left(T^{*}\right)=V_{c}(T)-\alpha\left(T^{*}-T\right)=V_{c}(T)-I V / I_{T} .
$$

The term $-I V / I_{T}$, with the constant $I_{T} \equiv(\alpha \kappa)^{-1}$, quantifies the self-heating effect and leads, with Eq. (4) inserted into Eq. (3), to a bias-current-dependent elevated temperature

$$
T^{*}=T+\frac{T_{c}-T}{I_{T} V_{c}(T)} I V .
$$

The larger $I_{T}$ the better is the heat transfer from the junction. By inserting Eq. (5) into Eq. (2) from the RSJ model, we obtain [19]

$$
I^{2} R^{2}-V^{2}= \begin{cases}{\left[V_{c}(T)-I V / I_{T}\right]^{2}} & \text { at } T^{*}<T_{c}, \\ 0 & \text { at } T^{*} \geq T_{c} .\end{cases}
$$

We used Eq. (7) for fitting our data. Despite the strong simplifications of the model, our hysteretic $I-V$ curves [JJ with $d_{\mathrm{Hf}-\mathrm{Ti}}=17 \mathrm{~nm}$ in Fig. 3(a) and JJs with $d_{\mathrm{Hf}-\mathrm{Ti}}=17$ and
$19.5 \mathrm{~nm}$ in Fig. 3(b)] are reasonably well described by the corresponding fits.

We note that a more precise quantitative description requires a detailed analysis of the junction dynamics in the presence of Joule heating, as, e.g., presented in Ref. [42]. This should include the modeling of the heat balance, as, e.g., given in Ref. [38], for the specific geometry of our junctions and should also take into account the correct $T$ dependence of $V_{c}(T)$ [43].

\section{NORMAL CONDUCTANCE BARRIER PROPERTIES}

The determination of the real barrier dimensions is essential for an accurate extraction of the JJ parameters like $j_{c}$, from which the coherence length $\xi_{N}$ in the normal conducting barrier can be estimated. We compare the experimentally determined specific JJ resistance

$$
r_{n}=R_{n} A,
$$

with

$$
r_{n}^{\prime}=\rho_{\mathrm{Hf}-\mathrm{Ti}} d_{\mathrm{Hf}-\mathrm{Ti}},
$$

calculated from the resistivity $\rho_{\mathrm{Hf}-\mathrm{Ti}}$ of Hf-Ti and the JJ barrier thickness $d_{\mathrm{Hf}-\mathrm{Ti}}$. Here, $R_{n}$ is obtained from the $I-V$ curves as the fitting parameter in the (modified) RSJ model (see Sec. III) and $A$ is the JJ area. Both quantities $r_{n}$ and $r_{n}^{\prime}$ should match within the experimental uncertainty if the JJ resistance is given by $\rho_{\mathrm{Hf}-\mathrm{Ti}} d_{\mathrm{Hf}-\mathrm{Ti}} / A$. This has been verified by measurements of $R_{n}$ of comparably large JJs with lateral dimensions greater than $400 \mathrm{~nm}$, for which one can neglect uncertainties in the JJ size. The value of $\rho_{\mathrm{Hf}-\mathrm{Ti}} \approx(105 \pm$ 3) $\mu \Omega \mathrm{cm}[44]$ has been extracted from measurements on a variety of Hf-Ti thin film stripes at $T=4.2 \mathrm{~K}$.

Some strict assumptions, however, should be valid for the correctness of this method. First, the thickness of the barrier material should be assumed $d_{\mathrm{Hf}-\mathrm{Ti}}=$ const over the whole JJ area. Additionally, the thin interface regions of $\mathrm{Nb}$ contacting the normal conducting layer and, therefore, experiencing penetration of quasiparticles due to the inverse proximity effect, should not contribute substantially to $r_{n}$. This effect is negligible, if the boundary condition $\sigma_{N} / \xi_{N} \ll \sigma_{S} / \xi_{S}$ or, equivalently, $1 /\left(\rho_{\mathrm{Hf}-\mathrm{Ti}} \xi_{N}\right) \ll$ $1 /\left(\rho_{S} \xi_{S}\right)$ is satisfied [19]. Here, $\sigma_{\mathrm{N}}=1 / \rho_{\mathrm{Hf}-\mathrm{Ti}}$ is the conductivity of the normal conducting layer and $\rho_{S}=1 / \sigma_{S}$ is the normal state resistivity of the superconducting layers. With a typical value $\rho_{S} \approx 3.8 \mu \Omega \mathrm{cm}$ for the resistivity of our $\mathrm{Nb}$ films at $T$ slightly above their transition temperature $T_{c}$, we estimate $\xi_{S} \approx 22 \mathrm{~nm}[45,46]$. Hence, with a sufficiently large $\xi_{N} \approx 4.9 \mathrm{~nm}$ (for the determination of $\xi_{N}$, see Sec. V) at $T=4.2 \mathrm{~K}$, the inverse proximity effect can be neglected to significantly affect $r_{n}$ of our JJs.

Second, on the linear branch of the $I-V$ curve the generated power should be insufficient to heat up the barrier 


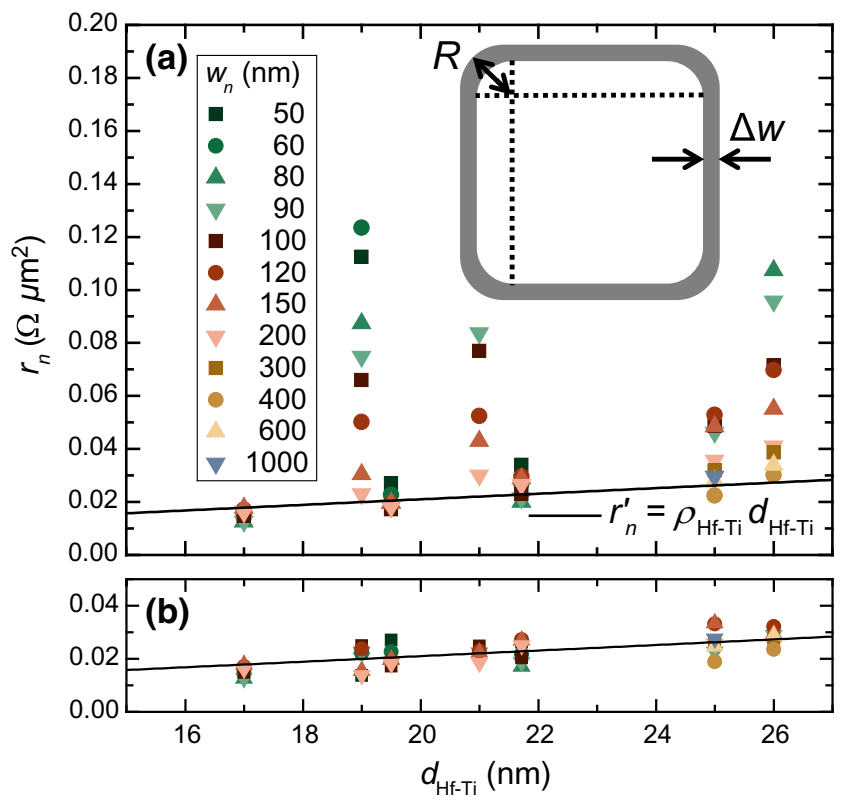

FIG. 4. Specific JJ resistance $r_{n}$ at $4.2 \mathrm{~K}$ versus the barrier thickness $d_{\mathrm{Hf}-\mathrm{Ti}}$ for JJs of different size. (a) Raw $r_{n}$ data, calculated with $A_{\text {geom }}$. (b) Values of $r_{n}$ recalculated with $A_{\text {eff }}$, considering some inactive region of width $\Delta w$ along the circumference of the JJs [indicated by the gray region in the inset of (a)].

significantly and thus increase $r_{n}$. Analysis of the shapes of the experimental $I-V$ curves shows that the resistance $R_{n}$ is almost independent of $I$ (see Sec. III). Hence, we conclude that a possible increase of $r_{n}$ due to heating is also negligible.

In Fig. 4 we show $r_{n}\left(d_{\mathrm{Hf}-\mathrm{Ti}}\right)$ for JJs of different barrier thickness and junction size. The raw measurement data [Fig. 4(a)] are determined via Eq. (8), using $A=A_{\text {geom }}$ for the $\mathrm{JJ}$ area evaluated by using SEM. For comparison, the expected linear behavior of $r_{n}^{\prime}\left(d_{\mathrm{Hf}-\mathrm{Ti}}\right)$ from Eq. (9) is shown as the solid line. While the data points for larger JJs are only slightly above the $r_{n}^{\prime}\left(d_{\mathrm{Hf}-\mathrm{Ti}}\right)$ line, smaller JJs tend to deviate more strongly. This behavior can be attributed to modifications of the barrier sidewalls, presumably caused by lateral damage during the fabrication process and oxidation. This results in a reduced effective area $A_{\text {eff, as }}$ compared to the geometric area $A_{\text {geom }}$.

To quantify the observed behavior, a crude JJ model can be introduced, which considers a complete exclusion of a small peripheral area of the barrier, with width $\Delta w$, from the electrical transport [gray region in the inset of Fig. 4(a)]. Obviously, the impact of this inactive region on the transport properties of the $\mathrm{JJ}$ is expected to become more pronounced as the nominal junction size becomes smaller. For all JJs, we take the same curvature radius $R=40 \mathrm{~nm}$. We then rescale the specific resistance of the JJs calculated with $A=A_{\text {eff }}\left(A_{\text {geom }}, \Delta w\right)$. Ideally, for a perfectly stable processing technology, one would expect to find the same value of $\Delta w$ for all wafer runs (which realize different values for $d_{\text {Hf-Ti }}$. However, our analysis shows that we can only obtain a good match of $R_{n} A_{\text {eff }}$ with $r_{n}^{\prime}$ if we assume different values of $\Delta w$ for different wafers (different $d_{\mathrm{Hf}-\mathrm{Ti}}$ ). We have to attribute this to uncontrolled variations of some fabrication conditions, which cause a variation of the edge damage for different wafers.

In Fig. 4(b) we show the rescaled specific resistance of the JJs, i.e., $r_{n}=R_{n} A_{\text {eff }}$ versus $d_{\mathrm{Hf}-\mathrm{Ti}}$. For JJs with the same barrier thickness (fabricated on the same wafer), a common value of $\Delta w$ is chosen to scale the average value of their specific resistance to $r_{n}^{\prime}\left(d_{\mathrm{Hf}-\mathrm{Ti}}\right)$. For the wafers with $d_{\mathrm{Hf}-\mathrm{Ti}}=19,21,25$, and $26 \mathrm{~nm}$, we obtain $\Delta w=26,24$, 19 , and $26 \mathrm{~nm}$, respectively, i.e., $\Delta w$ close to $20 \mathrm{~nm}$. For JJs with $d_{\mathrm{Hf}-\mathrm{Ti}}=21.7 \mathrm{~nm}$, we find a much smaller $\Delta w=3 \mathrm{~nm}$. And the $r_{n}$ values of JJs with $d_{\mathrm{Hf}-\mathrm{Ti}}=17$ and $19.5 \mathrm{~nm}$ have not been rescaled at all (i.e., $\Delta w=0$ ), as they are already very close to $r_{n}^{\prime}\left(d_{\mathrm{Hf}-\mathrm{Ti}}\right)$. Note also that the variance of $r_{n}$ for JJs on the same wafer, i.e., with the same $d_{\mathrm{Hf}-\mathrm{Ti}}$, as a function of $\Delta w$ is close to a minimum when the mean of $r_{n}\left(d_{\mathrm{Hf}-\mathrm{Ti}}\right)$ approaches $r_{n}^{\prime}\left(d_{\mathrm{Hf}-\mathrm{Ti}}\right)$, supporting the rescaling of the data.

\section{DEPENDENCE OF THE CRITICAL CURRENT DENSITY $\boldsymbol{j}_{c}$ AND THE CHARACTERISTIC VOLTAGE $V_{c}$ ON BARRIER THICKNESS}

The dependencies of $j_{c}$ and $V_{c}$ at $4.2 \mathrm{~K}$ on $d_{\mathrm{Hf}-\mathrm{Ti}}$ (from 17 to $26 \mathrm{~nm}$ ) for the junctions that were analyzed in Sec. IV are shown in Figs. 5(a) and 5(b), respectively. Here, to obtain the critical current density $j_{c}=I_{c} / A$, we take $A=A_{\text {eff }}$ from the model introduced in Fig. 4. Under the assumptions that our JJs satisfy (i) the dirty limit condition $l_{N} \ll \xi_{N}$ [47], (ii) the boundary condition $\sigma_{N} / \xi_{N} \ll \sigma_{\mathrm{S}} / \xi_{S}$ (see Sec. IV), and (iii) the long junction limit $d_{\mathrm{Hf}-\mathrm{Ti}} \gg$ $\xi_{N}=\left[\hbar D /\left(2 \pi k_{B} T\right)\right]^{1 / 2}$, with the diffusion coefficient $D=$ $v_{F} l_{N} / 3$ ( $v_{F}$ is the Fermi velocity and $l_{N}$ the mean-free path) and the Boltzmann constant $k_{B}$, the presented data can be fitted using the simplified expression [48]

$$
j_{c}=j_{c 0} \exp \left(-d_{\mathrm{Hf}-\mathrm{Ti}} / \xi_{N}\right)
$$

In Fig. 5(b) the data points are calculated from Eq. (1), where $I_{c}$ and $R_{n}$ are extracted as the fitting parameters within the framework of the standard [Eq. (2)] or extended [Eq. (7)] RSJ model applied to nonhysteretic or hysteretic $I-V$ curves, respectively. As long as the junction resistance $R_{n} \propto d_{\mathrm{Hf}-\mathrm{Ti}}$, the behavior of $V_{c}$ is expected to obey the relation

$$
V_{c}=V_{c 0} \frac{d_{\mathrm{Hf}-\mathrm{Ti}}}{\xi_{N}} \exp \left(-d_{\mathrm{Hf}-\mathrm{Ti}} / \xi_{N}\right)
$$

Despite the clear scatter in the data shown in Fig. 5, we find reasonably good agreement with theoretical predictions given by Eqs. (10) and (11) over a sufficiently 

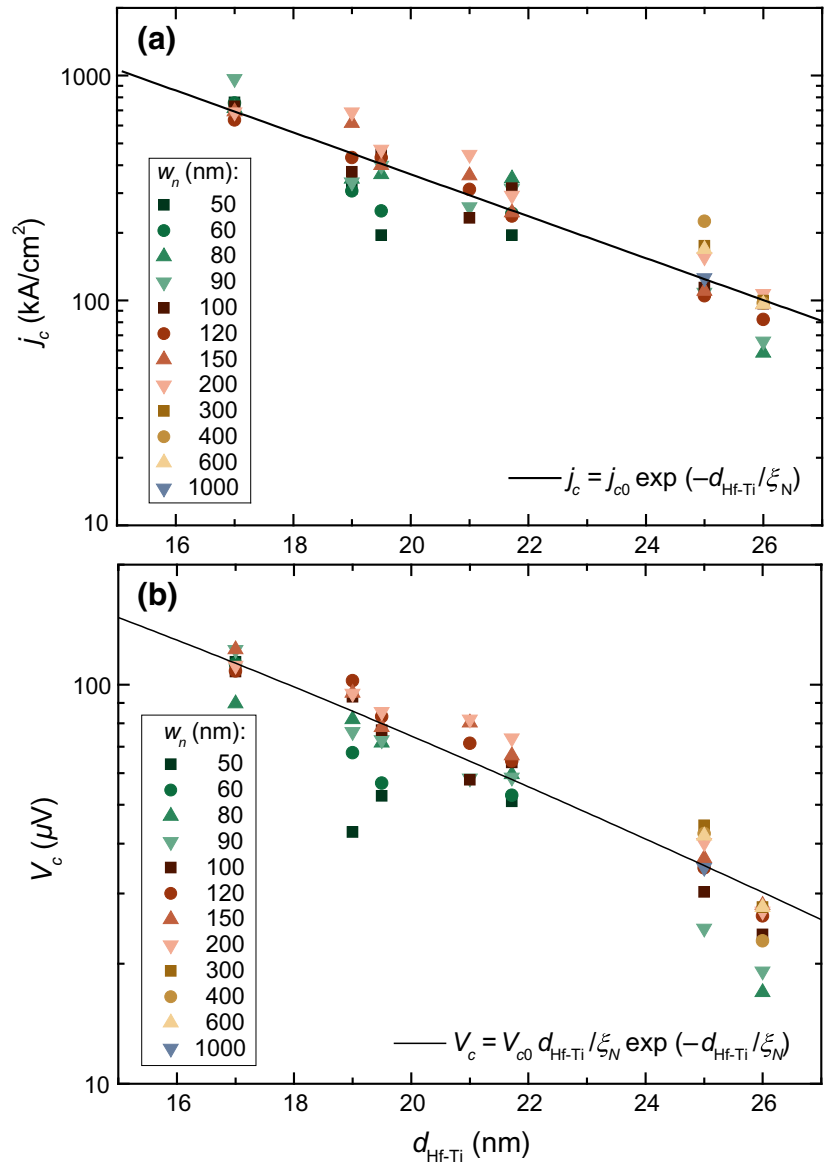

FIG. 5. Experimental data (symbols) of (a) the critical current density $j_{c}$ and (b) the characteristic voltage $V_{c}$, measured at $4.2 \mathrm{~K}$ versus the barrier thickness $d_{\mathrm{Hf}-\mathrm{Ti}}$. The solid lines are fits to the data, using Eq. (10) in (a) and Eq. (11) in (b).

wide range of $d_{\mathrm{Hf}-\mathrm{Ti}}$ with fitting parameters $j_{c 0}=(27 \pm$ 13) $\mathrm{MA} / \mathrm{cm}^{2}, V_{c 0}=(930 \pm 110) \mu \mathrm{V}$, and $\xi_{N}=(4.85 \pm$ $0.75) \mathrm{nm}$. We note that most of the data points for the smaller JJs in both plots lie below the fitted curve whereas the data for larger JJs are above the fit. This behavior may be related to some degradation of the barrier properties close to the inactive peripheral area. We also note that, from Eqs. (9)-(11), the relation $V_{c 0} /\left(j_{c 0} \xi_{N}\right)=$ $\rho_{\text {Hf-Ti }}$ follows. With the obtained fit parameters we calculate $V_{c 0} /\left(j_{c 0} \xi_{N}\right)=(71 \pm 37) \mu \Omega \mathrm{cm}$. The independently determined value for $\rho_{\mathrm{Hf}-\mathrm{Ti}}=(105 \pm 3) \mu \Omega \mathrm{cm}$ is at the upper limit, but still within the confidence interval for $V_{c 0} /\left(j_{c 0} \xi_{N}\right)$.

\section{NOISE PROPERTIES}

Within the RSJ model, the white noise of a JJ is considered by thermal Johnson-Nyquist noise of resistance $R_{n}$. In the case of a conventional SIS JJ design, $R_{n}$ is due to a separate metallic film (shunt resistor) located not in immediate proximity to the $\mathrm{JJ}$ and not necessarily extremely small, which therefore can be reasonably well thermalized. In SNS JJs, the barrier itself nicely conducts due to quasiparticles, and hence serves as the shunt resistor. Because of the rather small volume of the barrier and poor thermal conductance of the superconducting electrodes, the heat transfer from the barrier is not optimal and, as illustrated in the previous sections, can lead to the rise of the local electron (quasiparticle) temperature in the barrier. The consequence of this effect, thus, should not only be the appearance of hysteresis in the $I-V$ curves, but also some increase of thermal noise, as will be analyzed in the following.

In Fig. 6(a) we show four selected spectra of the rootmean-square (rms) spectral density of the voltage noise $S_{V}^{1 / 2}$ versus frequency $f$ at $T=4.2 \mathrm{~K}$, recorded at different $I$ values along the $I-V$ curve (Fig. 6(c), circles) of a $w_{n}=$ $50 \mathrm{~nm} \mathrm{JJ}$ with $d_{\mathrm{Hf}-\mathrm{Ti}}=17 \mathrm{~nm}$. The low-frequency part of those spectra shows the approximate scaling $S_{V} \propto 1 / f$ and will be discussed at the end of this section. Here, we focus on the white noise in those spectra. In Fig. 6(b) we show $S_{V}^{1 / 2}(I)$ (circles), obtained from six voltage noise spectra at frequency $f=100 \mathrm{kHz}$. This frequency is above the $1 / f$ corner (approximately 10-20 kHz) and, therefore, characterizes the white noise level of the JJ. The measurements are carried out at $T=4.2 \mathrm{~K}$ in a magnetically shielded environment making use of a two-stage cascade readout with a SQUID amplifier as described in Ref. [32]. The noise contribution of our measurement setup is verified to be negligible through measurements of the JohnsonNyquist noise of a stand-alone cold resistor $(R=1 \Omega)$ connected instead of a JJ.

For comparison with experimental white noise data, we calculate the rms voltage noise via

$$
S_{V}(I)=4 k_{B} T^{*}(I) \frac{R_{d}^{2}(I)}{R_{n}}\left[1+0.5\left(\frac{I_{c}\left(T^{*}\right)}{I}\right)^{2}\right]
$$

(with the differential resistance $R_{d} \equiv d V / d I$ ), which takes into account the contribution of the down-mixed highfrequency noise [the term proportional to $\left.\left(I_{c} / I\right)^{2}\right]$, as derived from the RSJ model $[49,50]$. To obtain the relevant quantities that enter into Eq. (12), we fitted the measured $I-V$ curve at $4.2 \mathrm{~K}$ to the extended model, Eq. (7), as shown in Fig. 6(c). In Fig. 6(b) we show the according fit to the experimental $R_{d}(I)$ curve. This fit yields $R_{n}=3 \Omega, I_{c}=40.6 \mu \mathrm{A}$ [i.e., $V_{c}(4.2 \mathrm{~K})=121.5 \mu \mathrm{V}$ ], $I_{T}=170.3 \mu \mathrm{A}$, and the dependence $R_{d}(I)$ that enters into Eq. (12). From Eq. (5) we obtain $I_{c}\left(T^{*}\right)=V_{c}\left(T^{*}\right) / R_{n}$. With $T_{c}=9.2 \mathrm{~K}$ we obtain $T^{*}(I)$ via Eq. (6), as shown in Fig. 6(c). From Eq. (4) we then obtain $\alpha=24.3 \mu \mathrm{V} / \mathrm{K}$, which gives $\kappa=\left(\alpha I_{T}\right)^{-1}=2.42 \times 10^{8}(\mathrm{~K} / \mathrm{W})$.

Obviously, the extended RSJ model predicts a significant increase of $T^{*}$ with $I$, which reaches up to $T_{c}$ within the bias current range shown in Fig. 6. The calculated $S_{V}(I)$ from Eq. (12) is shown in Fig. 6(b) as a thick solid 
(a)
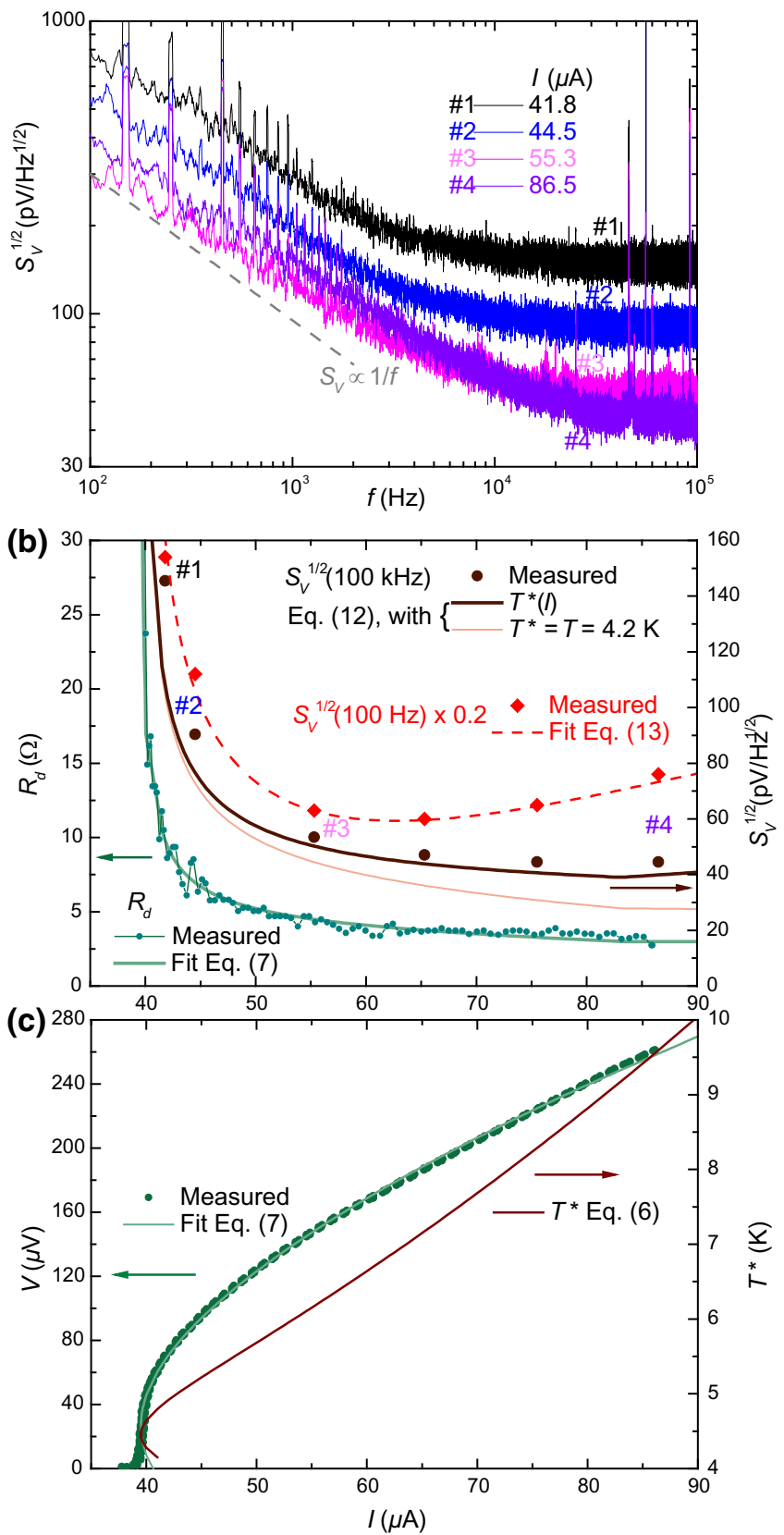

FIG. 6. Electric transport and noise properties of a $w_{n}=50$ $\mathrm{nm}$ JJ $\left(d_{\mathrm{Hf}-\mathrm{Ti}}=17 \mathrm{~nm}\right)$ measured at $T=4.2 \mathrm{~K}$. (a) rms voltage noise spectra $S_{V}^{1 / 2}(f)$ taken at four different values of the current $I$. The dashed line indicates $1 / f$ noise. (b) Differential resistance $R_{d}$ (small circles plus thin line) and rms voltage noise $S_{V}^{1 / 2}$ [labels nos. 1-4 refer to spectra in (a)] in the thermal white noise (circles) and $1 / f$ noise (diamonds) regimes versus the current $I \gtrsim I_{c}$. For comparison, solid thick lines show calculations based on the extended RSJ model Eqs. (7) and (12), with $T^{*}(I)$ due to self-heating (thick line). The solid thin line shows the calculated $S_{V}^{1 / 2}(I)$ if one neglects heating. The dashed line is a fit to the low $f$ data (both scaled by a factor of 0.2). (c) Measured (circles) and fitted (solid line) $I-V$ curves based on the extended RSJ model and effective temperature $T^{*}(I)$ calculated from Eq. (6). line. Despite the simplifying assumptions of the extended RSJ model, we find reasonably good agreement with the experimental data, which are systematically slightly above the calculation; however, the qualitative dependence of $S_{V}$ on $I$ is reproduced. For comparison, the thin solid line in Fig. 6(b) shows the calculated $S_{V}(I)$ assuming that $T^{*}=$ $T=4.2 \mathrm{~K}$, i.e., negligible self-heating, which yields an increasing deviation from the experimental noise data with increasing $I$.

We conclude that thermal imbalance induced by large dissipation inside the small volume of the Hf-Ti barrier leads to an elevated effective temperature $T^{*}$, which increases with bias current $I$, and which primarily causes the discrepancy between the experiment and the theory if self-heating is neglected.

Finally, we address the $1 / f$ noise, which is visible in the voltage noise spectra shown in Fig. 6(a) and clearly depends on the applied current $I$. To illustrate this, we include in Fig. 6(b) the measured $S_{V}^{1 / 2}(100 \mathrm{~Hz})$ versus $I$ (diamonds). Noise measurements on nano-SQUIDs based on our SNS JJs revealed $1 / f$ noise in the flux noise spectra, which could be suppressed by applying a bias reversal scheme. This indicates that the $1 / f$ noise is due to critical current and resistance fluctuations in our JJs $[32,34]$. To quantify the strength of those fluctuations in the single $\mathrm{JJ}$ discussed here, we follow the analysis described in Refs. [51,52], which yields, for the spectral density of the voltage noise of a single $\mathrm{JJ}$ in the $1 / f$-noise regime,

$S_{V}(f)=\left(V-R_{d} I\right)^{2} S_{i}(f)+V^{2} S_{r}(f)+k\left(V-R_{d} I\right) V S_{i r}(f)$.

Here, $S_{i}=S_{I_{c}} / I_{c}^{2}$ and $S_{r}=S_{R_{n}} / R_{n}^{2}$ are normalized spectral densities of critical current and resistance fluctuations, respectively, $S_{i r}=\sqrt{S_{I_{c}} S_{R_{n}}} /\left(I_{c} R_{n}\right)$ is the normalized crossspectral density of the fluctuations, and $k$ is a correlation factor, which can take values from -2 to +2 for fully antiphase and in-phase correlated $I_{c}$ and $R_{n}$ fluctuations, respectively [52]. The dashed line in Fig. 6(b) shows a fit of the $S_{V}^{1 / 2}(100 \mathrm{~Hz})$ data to Eq. (13). From the fit, we obtain the parameters $S_{i}(100 \mathrm{~Hz})=(5.2 \pm$ 1.4) $\times 10^{-12} / \mathrm{Hz}, S_{r}=(2.0 \pm 0.7) \times 10^{-12} / \mathrm{Hz}$, and $k=$ $(-0.1 \pm 1.1)$. The values obtained for $S_{i}$ and $S_{r}$ have the same order of magnitude, which means that $I_{c}$ and $R_{n}$ fluctuations have similar strength. The correlation factor $k$ is close to zero; however, due to the large error (95\% confidence interval), we cannot draw the conclusion that the fluctuations are uncorrelated.

For SIS Josephson junctions, the major source of $1 / f$ noise is attributed to $I_{c}$ and $R_{n}$ fluctuations that are caused by trapping and detrapping of charge carriers in a sufficiently large number of defects (localized electronic states) in the tunnel barrier [53]. For Nb- and Al-based SIS JJs with $\mathrm{AlO}_{x}$ barriers, empirical relations for $S_{i}$ and $S_{r} \propto$ 
$T^{2} /(A f)[54]$ or $\propto T /(A f)$ [55] have been found. Inserting numbers for our SNS JJ $\left(T=4.2 \mathrm{~K}, A=5 \times 10^{-3} \mu \mathrm{m}^{2}\right.$, $f=100 \mathrm{~Hz}$ ) into the relation given in Ref. [55] predicts $S_{i}=S_{r} \approx 0.8 \times 10^{-12} / \mathrm{Hz}$, which is not too far from our fit parameters obtained above and about 2 orders of magnitude lower than obtained with the relation given in Ref. [54]. However, the $1 / f$ noise data used in Refs. [54] and [55] refer to much larger JJs from several square micrometres to $0.1 \mu \mathrm{m}^{2}$. For SIS JJs with smaller area, the lowfrequency noise is described by the superposition of only one or a few Lorentzians, which indicates that only one or a few traps are dominating the noise. This is in strong contrast to the almost featureless $1 / f$ noise that we observe for our SNS JJ. Hence, although the strength of $I_{c}$ and $R_{n}$ fluctuations seems to be comparable to SIS JJs with tunnel barriers, the mechanism that causes those low-frequency fluctuations in our SNS JJs is unknown. Here, we note that a model for $I_{c}$ fluctuations based on a large density of Kondo states at subgap energies located at the SI interfaces in tunnel junctions has been proposed, which could explain a featureless $1 / f$ noise even in very small JJs [56]. It has also been argued that the spin of a Kondo trap may couple to both superconducting electrodes, altering the phase difference across the JJ, and hence leading to $I_{c}$ fluctuations [57]. However, it is unclear at the moment whether such a mechanism may also be relevant for SNS JJs.

\section{IMPLICATIONS FOR NANO-SQUID PERFORMANCE}

For the application of nano-SQUIDs to the detection of the magnetization reversal of individual MNPs, the spin sensitivity $S_{\mu}^{1 / 2}=S_{\Phi}^{1 / 2} / \phi_{\mu}$ (with units $\mu_{B} / \sqrt{\mathrm{Hz}} ; \mu_{B}$ is the Bohr magneton) is a figure of merit, as this quantifies the minimum number of spin flips that can be detected in a bandwidth of $1 \mathrm{~Hz}$. Obviously, to optimize $S_{\mu}^{1 / 2}$, one has to realize nano-SQUIDs with as low as possible spectral density of flux noise $S_{\Phi}$ and achieve as strong as possible coupling between the nano-SQUID and a MNP, quantified by the coupling factor $\phi_{\mu}$ (magnetic flux $\Phi$ per moment $\mu$ coupled to a SQUID by a MNP with magnetic moment $\mu$ ).

Regarding minimization of SQUID noise, we first consider the energy resolution $\varepsilon \equiv S_{\Phi} / 2 L$ in the thermal white noise limit for symmetric dc SQUIDs with inductance $L$ of the SQUID loop and dimensionless screening parameter $\beta_{L} \equiv 2 L I_{c} / \Phi_{0}\left(I_{c}\right.$ is the critical current per JJ). From numerical simulations based on the RCSJ model (with most practical flux bias close to $\Phi_{0} / 4$ ), a minimum in $\varepsilon\left(\beta_{L}\right)$ has been predicted at $\beta_{L, \mathrm{opt}} \approx 0.5$ as $\varepsilon_{\min } \equiv$ $\varepsilon\left(\beta_{L, \text { opt }}\right) \approx 3 \Phi_{0} k_{B} T / V_{c}$ [58-60]. A similar result can be obtained by extrapolation of the analytical formula Eq. (7.45a) of Ref. [61], derived in the framework of the RSJ model for small values of $\beta_{L}$ (less than or similar to 0.3 ). Clearly, $\varepsilon_{\min }$ can be reduced by making $V_{c}$ as large as possible and setting the flux bias point closer to $\Phi_{0} / 2[61,62]$.
For our $\mathrm{JJ}$ technology, a larger $V_{c}$ is achieved by choosing an as small as possible Hf-Ti barrier thickness. For the smallest $d_{\mathrm{Hf}-\mathrm{Ti}}=17 \mathrm{~nm}$ (i.e., $V_{c} \approx 0.1 \mathrm{mV}$ ) used in this work, we estimate $\varepsilon_{\min } \approx 5.4 h$ ( $h$ is the Planck constant) at $T=4.2 \mathrm{~K}$, with $\varepsilon_{\min }$ taken from numerical simulations.

The flux noise $S_{\Phi}=2 L \varepsilon$ can then be minimized by choosing $\beta_{L}=\beta_{L \text {,opt }}$ (i.e., $\varepsilon=\varepsilon_{\min }$ ) and choosing $L$ as small as possible. With $d_{\mathrm{Hf}-\mathrm{Ti}}=17 \mathrm{~nm}$ (and $j_{c 0}=$ $27 \mathrm{MA} / \mathrm{cm}^{2}$ and $\xi_{N}=4.85 \mathrm{~nm}$ ) inserted into Eq. (10), the condition $\beta_{L}=0.5$ yields the condition for the optimum effective lateral JJ size $w_{\text {eff }} \equiv \sqrt{A_{\text {eff }}} \approx 250 \mathrm{~nm} / \sqrt{L / \mathrm{pH}}$.

The microstriplike geometry of our nano-SQUIDs [33] (two $\mathrm{Nb}$ lines on top of each other, vertically connected by two JJs) allows for the realization of small SQUID inductance, which can be adjusted by the distance $d_{\mathrm{JJ}}$ between the JJs. So far, a typical $d_{\mathrm{JJ}}=600 \mathrm{~nm}$ yields $L \approx 1 \mathrm{pH}$ [33]. For fixed $L=1 \mathrm{pH}$ (and $d_{\mathrm{Hf}-\mathrm{Ti}}=17 \mathrm{~nm}$, $w_{\text {eff }}=250 \mathrm{~nm}$, i.e., $\beta_{L}=0.5$ ), we find an optimum $S_{\Phi}^{1 / 2} \approx$ $41 n \Phi_{0} / \sqrt{\mathrm{Hz}}$, predicted from numerical simulation. This is in the range of lowest values achieved so far for nanoSQUIDs at $4.2 \mathrm{~K}[9,14,21]$.

With the improved nanopatterning technology, demonstrated in this work, the realization of $\mathrm{Nb}$ lines with $100 \mathrm{~nm} \times 100 \mathrm{~nm}$ cross section is feasible. For a MNP placed at a $10 \mathrm{~nm}$ distance to the loop, our simulations of the coupling factor [63] yield $\phi_{\mu} \approx 13 n \Phi_{0} / \mu_{B}$. With $S_{\Phi}^{1 / 2} \approx 41 n \Phi_{0} / \sqrt{\mathrm{Hz}}$, predicted from numerical simulations for optimum $\beta_{L}$ and $L=1 \mathrm{pH}$, this results in an estimated optimum spin sensitivity of approximately $3 \mu_{B} / \sqrt{\mathrm{Hz}}$. Obviously, biasing the nano-SQUID in the voltage state, slightly above the critical current, will slightly increase the values for optimum rms flux noise and spin sensitivity due to self-heating.

The predicted optimum flux noise at $L=1 \mathrm{pH}$, however, requires a fairly large $\mathrm{JJ}$ size, which in turn favors hysteresis in the $I-V$ curves [cf. Fig. 3(b)]. In principle, $L$ can be reduced even further by reducing $d_{\mathrm{JJ}}$; however, this will require even larger $\mathrm{JJ}$ sizes to achieve optimum noise performance. Moreover, smaller $d_{\mathrm{JJ}}$ will also reduce the mutual inductance between one of the $\mathrm{Nb}$ lines and the SQUID, which will make flux feedback more difficult.

\section{CONCLUSIONS}

We present here an optimized fabrication technology for downscaling the lateral size of self-shunted Nb-based trilayer sandwich-type SNS Josephson junctions to below $100 \mathrm{~nm}$. The use of the high-resistivity material Hf-Ti as a normal conducting layer provides characteristic voltages $V_{c}$ up to above $100 \mu \mathrm{V}$ and high critical current densities $j_{c}$ approaching $10^{6} \mathrm{~A} / \mathrm{cm}^{2}$ at $T=4.2 \mathrm{~K}$ for the thinnest barrier with thickness $d_{\mathrm{Hf}-\mathrm{Ti}}=17 \mathrm{~nm}$, used in this study. There is certainly room for further increases in $V_{c}$ and $j_{c}$ with a further decrease in $d_{\text {Hf-Ti }}$. 
Because of the much higher $j_{c}$, as compared to trilayer SIS Josephson junctions with an insulating barrier, this SNS junction technology offers the opportunity to develop ultrasensitive strongly miniaturized nano-SQUIDs, which can be used for high-resolution SQUID microscopy and for the investigation of individual magnetic nanoparticles. For such nano-SQUID applications, constrictiontype junctions (Dayem bridges) in single-layer devices are commonly used. So far, sandwich-type junctions for nano-SQUID applications have suffered from their stronger sensitivity to external magnetic fields, simply related to the larger junction size, as compared to Dayem bridges. This problem can be solved by decreasing the SNS junction size and $\mathrm{Nb}$ linewidth with the technology presented here.

In comparison to Dayem bridges, trilayer SNS junctions offer the advantage of better tunability of their electric transport characteristics via proper choice of barrier thickness. Moreover, the electric transport and thermal white noise properties of these SNS junctions, and SQUIDs based on them, can be well described within the RSJ model, which allows one to optimize the SQUID performance based on numerical simulation studies. In contrast, the proper modeling of Dayem bridges is not well developed, and also their strong sensitivity to small variations in size significantly hamper SQUID optimization.

The increases in $j_{c}$ and $V_{c}$ upon decreasing $d_{\mathrm{Hf}-\mathrm{Ti}}$ cause self-heating of the junctions biased in the voltage state. This leads to hysteresis in the $I-V$ curves, which is detrimental to SQUID readout in the voltage state, and which is an issue for many nanoscale Josephson junctions, including Dayem bridges. The choice of a smaller JJ area, and concomitant reduction in $I_{c}$, results in reduced heating; this should also enable us to operate the nano-SQUIDs down to lower $T$ (which comes with an increase in $j_{c}$ ) before hysteresis sets in. In this paper, we have analyzed self-heating effects within a relatively simple extended RSJ model. Certainly, more efforts are required to efficiently improve heat removal from the junctions. A more detailed analysis of heating effects, including the proper temperature dependence of JJ properties, will be treated in a separate publication [43].

A so far unexplored issue has been $1 / f$ noise in SNS JJs and SQUIDs based on them. We have shown for one of our smallest SNS JJs that an almost featureless $1 / f$ noise due to critical current and resistance fluctuations is present, and that its magnitude is comparable to SIS JJs with $\mathrm{AlO}_{x}$ tunnel barriers. However, the microscopic origin of this $1 / f$ noise in our SNS JJ is unknown. With the JJ technology introduced here, one now can perform detailed studies on the level of $1 / f$ noise as a function of lateral JJ size, barrier thickness, and temperature, which may provide important information on the basic mechanism of $1 / f$ noise in SNS JJs.
Finally, we note that the advances in deep submicron SNS junction technology presented here offer the opportunity to make full use of the further advantages of SNS sandwich-type junctions for nano-SQUID applications. In particular, this multilayer technology provides the possibility to create much more complex and advanced nano-SQUID architectures, as, e.g., the three-dimensional vector nano-SQUID [33] or nano-SQUID susceptometers [34], including serial gradiometers and auxiliary components, such as gradiometric feedback loops gradiometric transformers and rf filters. This may significantly widen the application potential of nano-SQUIDs in the field of nanomagnetism and high-resolution magnetic imaging.

\section{ACKNOWLEDGMENTS}

The authors thank K. Störr, R. Wendisch, P. Hinze, and J. Felgner for their help throughout the wafer fabrication and support at the SEM equipment, and J. Kohlmann for fruitful discussions. B.M. acknowledges funding by the German Academic Scholarship Foundation. M.J.M.P. acknowledges funding by the Alexander von Humboldt Foundation. This work is supported by Deutsche Forschungsgemeinschaft (DFG) (Grants No. KI 698/3-1, 3-2, No. KO 1303/13-1, 13-2, No. SCHU 1950/5-1, and No. BE 6680/1-2) and the COST action NANOCOHYBRI (Grant No. CA 16218).

[1] John Clarke, Alex I. Braginski, eds., The SQUID Handbook Vol. I Fundamentals and Technology of SQUIDs and SQUID Systems (Wiley-VCH, Weinheim, 2004).

[2] John Clarke, Alex I. Braginski, eds., The SQUID Handbook Vol. II Applications of SQUIDs and SQUID Systems (Wiley-VCH, Weinheim, 2006).

[3] John Gallop, SQUIDs: Some limits to measurement, Supercond. Sci. Technol. 16, 1575 (2003).

[4] V. Bouchiat, Detection of magnetic moments using a nano-SQUID: Limits of resolution and sensitivity in nearfield SQUID magnetometry, Supercond. Sci. Technol. 22, 064002 (2009).

[5] W. Wernsdorfer, From micro- to nano-SQUIDs: Applications to nanomagnetism, Supercond. Sci. Technol. 22, 064013 (2009).

[6] C. Granata and A. Vettoliere, Nano superconducting quantum interference device: A powerful tool for nanoscale investigations, Phys. Rep. 614, 1 (2016).

[7] M. J. Martínez-Pérez and D. Koelle, NanoSQUIDs: Basics \& recent advances, Phys. Sci. Rev. 2, 20175001 (2017).

[8] A. Buchter, J. Nagel, D. Rüffer, F. Xue, D. P. Weber, O. F. Kieler, T. Weimann, J. Kohlmann, A. B. Zorin, E. Russo-Averchi, R. Huber, P. Berberich, A. Fontcuberta i Morral, M. Kemmler, R. Kleiner, D. Koelle, D. Grundler, and M. Poggio, Reversal Mechanism of an Individual Ni Nanotube Simultaneously Studied by Torque and SQUID Magnetometry, Phys. Rev. Lett. 111, 067202 (2013). 
[9] T. Schwarz, R. Wölbing, C. F. Reiche, B. Müller, M. J. Martínez-Pérez, T. Mühl, B. Büchner, R. Kleiner, and D. Koelle, Low-Noise $\mathrm{YBa}_{2} \mathrm{Cu}_{3} \mathrm{O}_{7}$ nano-SQUIDs for Performing Magnetization-Reversal Measurements on Magnetic Nanoparticles, Phys. Rev. Appl. 3, 044011 (2015).

[10] M. J. Martínez-Pérez, B. Müller, D. Schwebius, D. Korinski, R. Kleiner, J. Sesé, and D. Koelle, NanoSQUID magnetometry of individual cobalt nanoparticles grown by focused electron beam induced deposition, Supercond. Sci. Technol. 30, 024003 (2017).

[11] M. J. Martínez-Pérez, B. Müller, J. Lin, L. A. Rodriguez, E. Snoeck, R. Kleiner, J. Sesé, and D. Koelle, Magnetic vortex nucleation and annihilation in bi-stable ultra-small ferromagnetic particles, Nanoscale 12, 2587 (2020).

[12] L. Chen, W. Wernsdorfer, C. Lampropoulos, G. Christou, and I. Chiorescu, On-chip SQUID measurements in the presence of high magnetic fields, Nanotechnology 21, 405504 (2010).

[13] S. Mandal, T. Bautze, O. A. Williams, C. Naud, É. Bustarret, F. Omnès, P. Rodière, T. Meunier, C. Bäuerle, and L. Saminadayar, The diamond superconducting quantum interference device, ACS Nano 5, 7144 (2011).

[14] D. Vasyukov, Y. Anahory, L. Embon, D. Halbertal, J. Cuppens, L. Ne'eman, A. Finkler, Y. Segev, Y. Myasoedov, M. L. Rappaport, M. E. Huber, and E. Zeldov, A scanning superconducting quantum interference device with single electron spin sensitivity, Nat. Nanotechnol. 8, 639 (2013).

[15] T. Schwarz, J. Nagel, R. Wölbing, M. Kemmler, R. Kleiner, and D. Koelle, Low-noise nano superconducting quantum interference device operating in tesla magnetic fields, ACS Nano 7, 844 (2013).

[16] A. H. Miklich, D. Koelle, T. J. Shaw, F. Ludwig, D. T. Nemeth, E. Dantsker, J. Clarke, N. McN. Alford, T. W. Button, and M. S. Colclough, Low-frequency excess noise in $\mathrm{YBa}_{2} \mathrm{Cu}_{3} \mathrm{O}_{7-x}$ dc superconducting quantum interference devices cooled in static magnetic fields, Appl. Phys. Lett. 64, 3494 (1994).

[17] R. Wölbing, J. Nagel, T. Schwarz, O. Kieler, T. Weimann, J. Kohlmann, A. B. Zorin, M. Kemmler, R. Kleiner, and D. Koelle, $\mathrm{Nb}$ nano superconducting quantum interference devices with high spin sensitivity for operation in magnetic fields up to 0.5 T, Appl. Phys. Lett. 102, 192601 (2013).

[18] J. Nagel, A. Buchter, F. Xue, O. F. Kieler, T. Weimann, J. Kohlmann, A. B. Zorin, D. Rüffer, E. Russo-Averchi, R. Huber, P. Berberich, A. Fontcuberta i Morral, D. Grundler, R. Kleiner, D. Koelle, M. Poggio, and M. Kemmler, Nanoscale multifunctional sensor formed by a Ni nanotube and a scanning $\mathrm{Nb}$ nanoSQUID, Phys. Rev. B 88, 064425 (2013).

[19] K. K. Likharev, Superconducting weak links, Rev. Mod. Phys. 51, 101 (1979).

[20] L. Fritzsch, H. Elsner, M. Schubert, and H.-G. Meyer, SNS and SIS Josephson junctions with dimensions down to the submicron region prepared by a unified technology, Supercond. Sci. Technol. 12, 880 (1999).

[21] M. Schmelz, A. Vettoliere, V. Zakosarenko, N. De Leo, M. Fretto, R. Stolz, and C. Granata, 3D nanoSQUID based on tunnel nano-junctions with an energy sensitivity of $1.3 \mathrm{~h}$ at 4.2 K, Appl. Phys. Lett. 111, 032604 (2017).
[22] A. W. Kleinsasser, R. E. Miller, W. H. Mallison, and G. B. Arnold, Observation of Multiple Andreev Reflections in Superconducting Tunnel Junctions, Phys. Rev. Lett. 72, 1738 (1994).

[23] D. Hagedorn, O. Kieler, R. Dolata, R. Behr, F. Müller, J. Kohlmann, and J. Niemeyer, Modified fabrication of planar sub- $\mu \mathrm{m}$ superconductor-normal metal-superconductor Josephson junctions for use in a Josephson arbitrary waveform synthesizer, Supercond. Sci. Technol. 19, 294 (2006).

[24] B. Baek, P. D. Dresselhaus, and S. P. Benz, Cosputtered amorphous $\mathrm{Nb}_{x} \mathrm{Si}_{1-x}$ barriers for Josephsonjunction circuits, IEEE Trans. Appl. Supercond. 16, 1966 (2006).

[25] F. Müller, T. Scheller, R. Wendisch, R. Behr, O. Kieler, L. Palafox, and J. Kohlmann, NbSi barrier junctions tuned for metrological applications up to $70 \mathrm{GHz}: 20 \mathrm{~V}$ arrays for programmable Josephson voltage standards, IEEE Trans. Appl. Supercond. 23, 1101005 (2013).

[26] W. C. Stewart, Current-voltage characteristics of Josephson junctions, Appl. Phys. Lett. 12, 277 (1968).

[27] D. E. McCumber, Effect of ac impedance on dc voltagecurrent characteristics of superconductor weak-link junctions, J. Appl. Phys. 39, 3113 (1968).

[28] L. G. Aslamazov, A. I. Larkin, and Yu. N. Ovchinnikov, Josephson effect in superconductors separated by a normal metal, Sov. Phys. JETP 28, 171 (1969), Zh. Eksp. Teor. Fiz. 55, 323-335 (1969).

[29] D. Hazra, L. M. A. Pascal, H. Courtois, and A. K. Gupta, Hysteresis in superconducting short weak links and $\mu$-SQUIDs, Phys. Rev. B 82, 184530 (2010).

[30] N. Kumar, C. B. Winkelmann, S. Biswas, H. Courtois, and A. K. Gupta, Controlling hysteresis in superconducting constrictions with a resistive shunt, Supercond. Sci. Technol. 28, 072003 (2015).

[31] J. Nagel, O. F. Kieler, T. Weimann, R. Wölbing, J. Kohlmann, A. B. Zorin, R. Kleiner, D. Koelle, and M. Kemmler, Superconducting quantum interference devices with submicron $\mathrm{Nb} / \mathrm{HfTi} / \mathrm{Nb}$ junctions for investigation of small magnetic particles, Appl. Phys. Lett. 99, 032506 (2011).

[32] J. Beyer, M. Klemm, J. H. Storm, O. Kieler, T. Weimann, and V. Morosh, Noise of dc-SQUIDs with planar submicrometer $\mathrm{Nb} / \mathrm{HfTi} / \mathrm{Nb}$ junctions, Supercond. Sci. Technol. 28, 085011 (2015).

[33] M. J. Martínez-Pérez, D. Gella, B. Müller, V. Morosh, R. Wölbing, J. Sesé, O. Kieler, R. Kleiner, and D. Koelle, Three-axis vector nano superconducting quantum interference device, ACS Nano 10, 8308 (2016).

[34] S. Bechstein, C. Köhn, D. Drung, J.-H. Storm, O. Kieler, V. Morosh, and T. Schurig, Investigation of nanoSQUID designs for practical applications, Supercond. Sci. Technol. 30, 034007 (2017).

[35] O. F. Kieler, R. Iuzzolino, and J. Kohlmann, Sub- $\mu$ m SNS Josephson junction arrays for the Josephson arbitrary waveform synthesizer, IEEE Trans. Appl. Supercond. 19, 230 (2009).

[36] V. N. Gubankov, K. K. Likharev, and N. M. Margolin, Properties of superconducting point contacts, Sov. Phys. Solid State 14, 819 (1972), Fiz. Tverd. Tela 14, 953-960 (1972). 
[37] W. J. Skocpol, M. R. Beasley, and M. Tinkham, Selfheating hotspots in superconducting thin-film microbridges, J. Appl. Phys. 45, 4054 (1974).

[38] H. Courtois, M. Meschke, J. T. Peltonen, and J. P. Pekola, Origin of Hysteresis in a Proximity Josephson Junction, Phys. Rev. Lett. 101, 067002 (2008).

[39] Y. Song, Origin of "capacitance" in superconducting microbridges, J. Appl. Phys. 47, 2651 (1976).

[40] A. Baratoff and L. Kramer, in Proc. of the Internat. Conf. on Superconducting Quantum Interference Devices and their Applications, edited by H. D. Hahlbohm and H. Lübbig (Walter de Gruyter, New York, 1977), p. 51.

[41] L. Angers, F. Chiodi, G. Montambaux, M. Ferrier, S. Guéron, H. Bouchiat, and J. C. Cuevas, Proximity dc squids in the long-junction limit, Phys. Rev. B 77, 165408 (2008).

[42] A. K. Gupta, N. Kumar, and S. Biswas, Temperature and phase dynamics in superconducting weak-link, J. Appl. Phys. 116, 173901 (2014).

[43] J. Linek, R. Varga, K. Wurster, B. Müller, V. Morosh, T. Weimann, O. Kieler, R. Kleiner, and D. Koelle, Selfheating effects in SNS Nb-HfTi-Nb sub- $\mu \mathrm{m}$ Josephson junctions (unpublished).

[44] J. Felgner, O. F. Kieler, J. Schurr, J. Herick, S. Bauer, R. Wendisch, and K. Störr, Evaluation of material parameters for on-chip capacitors and resistors for superconducting circuits, Poster presentation at Kryoelektronische Bauelemente 2017, Bad Aibling (Germany).

[45] M. Tinkham, Introduction to Superconductivity (Dover Publications, Inc., Mineola, N.Y., 2004).

[46] A. F. Mayadas, R. B. Laibowitz, and J. J. Cuomo, Electrical characteristics of rf-sputtered single-crystal Niobium films, J. Appl. Phys. 43, 1287 (1972).

[47] P. G. De Gennes, Boundary effects in superconductors, Rev. Mod. Phys. 36, 225 (1964).

[48] K. A. Delin and A. W. Kleinsasser, Stationary properties of high-critical-temperature proximity effect Josephson junctions, Supercond. Sci. Technol. 9, 227 (1996).

[49] K. K. Likharev and V. K. Semenov, Fluctuation spectrum in superconducting point junctions, JETP Lett. 15, 442 (1972), ZhETF Pis. Red. 15, 625-629 (1972).

[50] R. H. Koch, D. J. Van Harlingen, and J. Clarke, QuantumNoise Theory for the Resistively Shunted Josephson Junction, Phys. Rev. Lett. 45, 2132 (1980).
[51] A. H. Miklich, J. Clarke, M. S. Colclough, and K. Char, Flicker $(1 / f)$ noise in biepitaxial grain boundary junctions of $\mathrm{YBa}_{2} \mathrm{Cu}_{3} \mathrm{O}_{7-x}$, Appl. Phys. Lett. 60, 1899 (1992).

[52] A. Marx, U. Fath, W. Ludwig, R. Gross, and T. Amrein, $1 / f$ noise in $\mathrm{Bi}_{2} \mathrm{Sr}_{2} \mathrm{CaCu}_{2} \mathrm{O}_{8+x}$ bicrystal grain-boundary Josephson junctions, Phys. Rev. B 51, 6735 (1995).

[53] C. T. Rogers and R. A. Buhrman, Composition of $1 / f$ Noise in Metal-Insulator-Metal Tunnel Junctions, Phys. Rev. Lett. 53, 1272 (1984).

[54] D. J. Van Harlingen, T. L. Robertson, B. L. T. Plourde, P. A. Reichardt, T. A. Crane, and J. Clarke, Decoherence in Josephson-junction qubits due to critical-current fluctuations, Phys. Rev. B 70, 064517 (2004).

[55] C. D. Nugroho, V. Oryanchik, and D. J. Van Harlingen, Low frequency resistance and critical current fluctuations in Al-based Josephson junctions, Appl Phys. Lett. 102, 142602 (2013).

[56] L. Faoro and L. B. Ioffe, Microscopic origin of critical current fluctuations in large, small, and ultra-small area Josephson junctions, Phys. Rev. B 75, 132505 (2007).

[57] L. Faoro, A. Kitaev, and L. B. Ioffe, Quasiparticle Poisoning and Josephson Current Fluctuations Induced by Kondo Impurities, Phys. Rev. Lett. 101, 247002 (2008).

[58] C. D. Tesche and J. Clarke, DC SQUID: Noise and optimization, J. Low Temp. Phys. 29, 301 (1977).

[59] J. J. P. Bruines, V. J. de Waal, and J. E. Mooij, Comment on: "Dc SQUID: Noise and optimization" by Tesche and Clarke, J. Low Temp. Phys. 46, 383 (1982).

[60] B. Chesca, R. Kleiner, and D. Koelle, in The SQUID Handbook, Vol. I: Fundamentals and Technology of SQUIDs and SQUID systems, edited by John Clarke and Alex I. Braginski (Wiley-VCH, Weinheim, 2004), Chap. 2, p. 29.

[61] K. K. Likharev, Dynamics of Josephson Junctions and Circuits (Gordon and Breach Science Publishers, New York, 1986).

[62] V. V. Danilov, K. K. Likharev, and O. V. Snigirev, in SQUID'80: Superconducting Quantum Interference Devices and their Applications, edited by H.D. Hahlbohm and H. Luebbig (W. de Gruyter, Berlin, 1980), p. 473.

[63] R. Wölbing, T. Schwarz, B. Müller, J. Nagel, M. Kemmler, R. Kleiner, and D. Koelle, Optimizing the spin sensitivity of grain boundary junction nanoSQUIDs - towards detection of small spin systems with single-spin resolution, Supercond. Sci. Technol. 27, 125007 (2014). 